\title{
Exact Solutions for the Elastic Buckling Problem of Moderately Thick Beams
}

\author{
Hyginus N. Onah ${ }^{1}$, Clifford U. Nwoji ${ }^{1}$, Michael E. Onyia ${ }^{1}$, Benjamin O. Mama ${ }^{1}$, Charles C. Ike ${ }^{2 *}$ \\ ${ }^{1}$ Department of Civil Engineering, University of Nigeria, Nsukka 410001, Enugu State, Nigeria \\ ${ }^{2}$ Department of Civil Engineering, Faculty of Engineering, Enugu State University of Science and Technology, Enugu 400001, \\ Enugu State, Nigeria
}

Corresponding Author Email: charles.ike@esut.edu.ng

https://doi.org/10.18280/rcma.300205

Received: 15 June 2019

Accepted: 24 January 2020

\author{
Keywords: \\ first order shear deformation theory, Euler- \\ Bernoulli beam theory, elastic buckling \\ problem, critical buckling load, thick beam
}

\begin{abstract}
We present the elastic buckling problem of moderately thick and thick beams as a boundary value problem of the classical mathematical theory of elasticity. The study considered homogeneous, isotropic, linear elastic beams. Small deformation assumptions were used together with kinematic, constitutive relations and the differential equations of equilibrium to obtain the governing field equations as a fourth order non-homogeneous ordinary differential equation (ODE) when both axial, compressive and transverse loads are considered, and a fourth order homogeneous ODE when only axial compressive force is considered. Using trial function method, the homogeneous ODE is solved in general for any end support conditions to obtain a general solution for the buckled beam in terms of four unknown constants of integration. The boundary conditions corresponding to the four cases of end support conditions considered were used to obtain the characteristic buckling equations, which were expanded to obtain transcendental equations with an infinite number of roots in each case, thus yielding an infinite number of buckling loads. The least root of the transcendental equations was used to obtain the critical buckling load, which was found to depend on the ratio $h / l$ and the Poisson's ratio, $\mu$. Critical buckling loads for each end support condition was calculated and tabulated. The results show that for each end support condition, as $h / l<0.02$, the critical buckling load coefficient obtained was approximately equal to the critical buckling load coefficient of Euler - Bernoulli beam. As $h / l>0.02$, which is the threshold for thin beams, the critical buckling load is found to be much smaller than the critical buckling load obtained from Euler - Bernoulli theory. It is thus concluded that the shear deformable theory is necessary for a more realistic analysis of the critical load buckling capacities of moderately thick, and thick beams for safety in their design.
\end{abstract}

\section{INTRODUCTION}

Elastic buckling analysis of thick beams are fundamentally two dimensional problems of the mathematical theory of elasticity and are formulated and solved using the tools and analytical methods. The simplification of elastic buckling problem of thick beams from a two dimensional problem of the theory of elasticity to one dimensional approximate problems have always been the focus and objective of researchers who worked in the area of thick beam stability [1, 2].

Various beam theories have been developed in the literature for the problem of beam buckling. The classical Euler Bernoulli beam theory (EBT) was formulated using the Euler - Bernoulli hypothesis that the plane cross-sections initially orthogonal to the neutral axis before deformation would remain plane and orthogonal to the neutral axis after deformation $[3,4]$. The Euler - Bernoulli theory has been used in the literature to model flexural, dynamic and stability analysis and behaviours of beams. The Euler-Bernoulli hypothesis implies that the effects of transverse shear deformation are ignored, thus effectively limiting the scope and applicability of the EBT to slender (thin) beams where transverse shear deformation effects contribute negligibly to the overall behaviour. The limitations of the classical EBT have been observed for flexural, dynamic and stability problems of thick and moderately thick beams.

The EBT underestimates the values of displacements and overestimates the frequencies and buckling loads of thick and moderately thick beams where shear deformation effects become significant in the flexural, dynamic and buckling behaviours of such beams. Other models and theories of beams were developed to address the shortcomings of the EBT [5]. The Timoshenko beam theory (TBT) which is a first order shear deformation theory (FSDT) for beams was formulated on the hypothesis that the plane cross-sections that are initially orthogonal to the neutral axis of the beam before deformation would remain plane but not necessarily orthogonal to the neutral axis after deformation $[6,7]$.

First order shear deformation theory thus involves a relaxation of the normality condition. The theory considers the linear variation of mid plane displacement. The theory considers that the transverse shear strain distribution is constant through the beam thickness, and thus violates the shear stress free boundary conditions on the top and bottom surfaces of the beam. The theory thus requires problem 
dependent shear stress correction/modification factors in order to appropriately represent the strain energy of deformation [810]. Timoshenko beam theory and other FSDT of beams are formulated in terms of two unknown functions.

The limitations and inadequacies of both the EBT, and the FSDT for the analysis of beam flexure, dynamics and buckling inspired the formulation and development of higher order shear deformation theories for the analysis of thick and moderately thick beams that would adequately account for the effect of transverse shear deformation, and also appropriately satisfy the shear stress free boundary conditions on the beam top and bottom surfaces.

Levinson [11], Krishna Murty [12], Heylinger and Reddy [13] and many other researchers have presented parabolic shear deformation theories for the analysis of thick and moderately thick beams under flexure, dynamics and buckling conditions.

Ghugal [10] extended the parabolic shear deformation theory of thick and moderately thick beams to include transverse normal strains and transverse shear strain effects for the flexure, dynamics and buckling analysis of isotropic thick beams. Ghugal and Shimpi [8] formulated and presented a trigonometric shear deformation theory that accounts for transverse shear deformation effects and which is thus applicable for the analysis of the flexural, dynamic and buckling behaviours of thick and moderately thick beams.

Sayyad and Ghugal [14] formulated trigonometric shear and normal deformation theory that considered the effects of transverse shear and normal deformations for the flexure of thick and moderately thick isotropic and laminated beams.

Soldatos [15] proposed a hyperbolic shear deformation theory for the flexural, dynamic and buckling analysis of thick and moderately thick isotropic, homogeneous beams under a variety of support conditions. Soldatos' theory was extended by Ghugal and Sharma [16].

Karama et al. [17] developed an exponential shear deformation theory for the analysis of thick and moderately thick beams under flexure, vibration and buckling and for different support conditions.

Sayyad [18] presented a unified beam theory [UBT] for the flexure, eigen frequency and stability analysis of thick and moderately thick beams. In the UBT, parabolic, trigonometric, hyperbolic, and exponential functions are used in terms of the thickness coordinates to represent the effect of transverse shear deformation, rendering the theory suitable for the modelling of thick and moderately thick beams.

Elastic buckling of beams is a failure situation where the beam is no longer able to carry lateral loads when the axially applied compressive force attains a critical value. The ratio of beam thickness to length and shear deformation effects have been known to affect the critical buckling loads since nondimensional critical buckling load reduces with increase in the ratio of beam thickness-to-length.

Unlike the TBT and FSDT that assume a constant transverse shear strain variation through the beam thickness, and hence require shear correction factors, HSDT of beams assume more realistic polynomial distribution of transverse shear strain through the beam thickness.

Despite the merits of the FSDT and HSDT in suitably describing /and modelling thick and moderately thick beams, they are generally governed by a system of coupled differential equations which increases the analytical rigours involved in their solution. Their governing equations involve increased number of independent unknown displacements and their solution require the specification of increased number of boundary conditions.

In this work, the problem of elastic buckling of a homogeneous isotropic beam of prismatic cross-section is derived and presented from fundamental principles as a boundary value problem of the theory of elasticity. The BVP is then solved in closed form to obtain exact solutions for different end support conditions.

\section{THEORETICAL FORMULATION OF THE THICK BEAM BUCKLING PROBLEM}

\subsection{Thick beam considered}

The thick beam considered in this study as shown in Figure 1 is defined by the system of three dimensional (3D) Cartesian coordinates:

$$
0 \leq x \leq l ;-b / 2 \leq y \leq b / 2 ;-h / 2 \leq z \leq h / 2
$$

where, $x, y, z$ are the 3D Cartesian coordinates $l$ is the length of the beam, $b$ is the width and $h$ is the total depth (thickness) of the beam. The longitudinal domain is defined by the $x$ coordinate variable while the cross-sectional dimensions are defined by the $y z$ coordinates. The thick beam can be submitted to any loading and boundary (support) conditions.



Figure 1. Thick beam buckling problem considered

\subsection{Fundamental assumptions}

The fundamental assumptions of the formulation are as follows:

(i) The inplane displacement field $u$ is decomposable into a displacement component analogous to the pure bending displacement in the classical Euler - Bernoulli beam bending theory and a displacement component attributable to shear deformation.

(ii) The transverse displacement field component in the $z$ coordinate direction is a function of the longitudinal coordinate position only.

(iii) Stress - strain relations are one dimensional.

(iv) The displacements are infinitesimally small compared to the beam thickness, resulting in strains that are considered infinitesimal

(v) body forces are ignored but can be accounted for by the inclusion of external forces.

(vi) The transverse displacement $w(x, z)$ is made of two components namely flexural component $w_{b}(x)$ and shear component, $w_{s}(x)$

(vii) The beam material is homogeneous, linearly elastic and isotropic.

\subsection{Displacement field}

The displacement field components are: 


$$
\begin{gathered}
u(x, z)=u(x)+z \varphi(x) \\
v(x, z)=0 \\
w(x, z)=w(x)=w_{b}(x)+w_{s}(x)
\end{gathered}
$$

where, $\varphi(x)$ is the rotation of the cross-section at the neutral axis; $u(x, z)$ is the displacement of the beam centre line in the $x$ direction; $v(x, z)$ is the displacement field in the $y$ direction; $w(x, z)$ is the transverse displacement of the beam centreline in the $z$ direction; $u(x)$ is the axial displacement according to the classical Euler-Bernoulli linear bending theory which is linear through the beam thickness; $z \varphi(x)$ is the displacement due to the transverse shear deformation which is nonlinear through the beam thickness.

The rotation $\varphi(x)$ is

$$
\varphi(x)=-\frac{d w_{b}(x)}{d x}
$$

where, $w_{b}$ is the transverse deflection due to bending. Hence, Equation (1) becomes:

$$
u(x, z)=u(x)-z \frac{d w_{b}(x)}{d x}
$$

\subsection{Strain-displacement relations}

The strain - displacement relations are:

$$
\begin{gathered}
\varepsilon_{x x}=\frac{\partial u}{\partial x}=\frac{\partial}{\partial x}\left(u(x)-z \frac{d w_{b}(x)}{d x}\right) \\
=\frac{\partial u(x)}{\partial x}-z \frac{\partial^{2} w_{b}(x)}{d x^{2}} \\
\varepsilon_{y y}=\frac{\partial v(x, z)}{\partial y}=0 \\
\varepsilon_{z z}=\frac{\partial w}{\partial z}=0 \\
\gamma_{x y}=\frac{\partial u(x, y)}{\partial y}+\frac{\partial v(x, z)}{\partial x}=0 \\
\gamma_{y z}=\frac{\partial v(x, z)}{\partial z}+\frac{\partial w(x, z)}{\partial y}=0 \\
\gamma_{x z}=\frac{\partial u(x, z)}{\partial z}+\frac{\partial w(x, z)}{\partial x}=\frac{d w_{s}(x)}{d x}
\end{gathered}
$$

where, $\varepsilon_{x x}, \varepsilon_{y y}, \varepsilon_{z z}$ are normal strains, $\gamma_{x y}, \gamma_{y z}$ and $\gamma_{x z}$ are shear strains.

\subsection{Stress-strain relations}

The one - dimensional stress - strain relations are:

$$
\begin{gathered}
\sigma_{x x}=E \varepsilon_{x x}=E \frac{d u(x)}{d x}-\left(E z \frac{d^{2} w_{b}(x)}{d x^{2}}\right) \\
\tau_{x z}=G \gamma_{x z}=G \frac{d w_{s}(x)}{d x}
\end{gathered}
$$

where, $E$ is the Young's modulus of elasticity $G$ is the shear modulus or modulus of rigidity.

\subsection{Stress resultants}

The bending moment $M(x)$ is:

$$
M_{x x}=\iint_{R^{2}} \sigma_{x x} z d y d z
$$

$R^{2}$ is the two dimensional region of integration. For beam column and beam buckling problems.

$$
M_{x x}=\iint_{R^{2}}-E z^{2} \frac{d^{2} w_{b}(x)}{d x} d y d z=-E I \frac{d^{2} w_{b}(x)}{d x^{2}}
$$

where, $I$ is the moment of inertia,

$$
I=\iint_{R^{2}} z^{2} d y d z=\int_{-h / 2}^{h / 2} \int_{-b / 2}^{b / 2} z^{2} d y d z
$$

The shear force $Q_{x x}$ is

$$
Q_{x}=\iint \tau_{x z} d A=\iint_{R^{2}} G \frac{d w_{s}(x)}{d x} d y d z=G b h \frac{d w_{s}(x)}{d x}
$$

Introducing a shear correction/modification factor, $k$, shear force is

$$
Q_{x}=k G h b \frac{d w_{s}(x)}{d x}=k G A \frac{d w_{s}(x)}{d x}
$$

\subsection{Differential equations of equilibrium}

The differential equation of equilibrium in the $x$ direction is

$$
\frac{\partial \sigma_{x x}}{\partial x}+\frac{\partial \tau_{x y}}{\partial y}+\frac{\partial \tau_{x z}}{\partial z}+f_{x}=0
$$

where, $f_{x}$ is the body force component in the $x$ direction.

For the thick beam problem, when body force $f_{x}$ is disregarded, the differential equation of equilibrium becomes:

$$
\frac{\partial \sigma_{x x}}{\partial x}+\frac{\partial \tau_{x z}}{\partial z}=0
$$

From this, we have:

$$
\iint_{R^{2}}\left(\frac{\partial \sigma_{x x}}{\partial x}+\frac{\partial \tau_{x z}}{\partial z}\right) z d y d z=0
$$


Hence,

$$
\begin{gathered}
\frac{\partial}{\partial x} \iint_{R^{2}} \sigma_{x x} z d y d z+\iint_{R^{2}} \tau_{x z} d y d z=0 \\
\frac{d}{d x} M_{x x}-Q_{x}=0
\end{gathered}
$$

where,

$$
\iint_{R^{2}} \tau_{z x} d y d z=Q_{x}
$$

and

$$
\tau_{x z}=-\tau_{z x}
$$

Hence by substitution,

$$
\frac{d}{d x}\left(-E I \frac{d^{2} w_{b}(x)}{d x^{2}}\right)-Q_{x}=0
$$

Rearranging,

$$
-\frac{d}{d x}\left(E I \frac{d^{2} w_{b}(x)}{d x^{2}}\right)=Q_{x}=k A G \frac{d w_{s}(x)}{d x}
$$

Re-expressing Eq. (27) we have:

$$
\frac{d w_{s}(x)}{d x}=-\frac{1}{k A G} \frac{d}{d x}\left(E I \frac{d w_{b}(x)}{d x}\right)
$$

Integrating Eq. (28) with respect to $x$, we have:

$$
\begin{gathered}
\int \frac{d w_{s}(x)}{d x} d x=w_{s}(x)=\int-\frac{1}{k A G} \frac{d}{d x}\left(E I \frac{d w_{s}(x)}{d x} d x\right) \\
w_{s}(x)=-\frac{1}{k A G} E I \frac{d^{2} w_{b}(x)}{d x^{2}}
\end{gathered}
$$

But

$$
G=\frac{E}{2(1+\mu)}
$$

$w_{s}(x)=\frac{-E I}{k A(E / 2(1+\mu))} \frac{d^{2} w_{b}(x)}{d x^{2}}=\frac{-2(1+\mu) I}{k A} \frac{d^{2} w_{b}(x)}{d x^{2}}$

For rectangular cross-sections,

$$
\begin{gathered}
A=b h \\
I=\frac{b h^{3}}{12} \\
k=\frac{5}{6}
\end{gathered}
$$

then,

$$
w_{s}(x)=\frac{-h^{2}(1+\mu)}{5} \frac{d^{2} w_{b}(x)}{d x^{2}}=\frac{-h^{2}(1+\mu)}{6 k} \frac{d^{2} w_{b}(x)}{d x^{2}}
$$

For circular cross-sections, of diameter, $d$ :

$$
\begin{gathered}
A=\frac{\pi d^{2}}{4} \\
I=\frac{\pi d^{4}}{64}
\end{gathered}
$$

then,

$$
\begin{aligned}
w_{s}(x) & =\frac{-2(1+\mu) \frac{\pi d^{4}}{64}}{k \frac{\pi d^{2}}{4}} \frac{d^{2} w_{b}(x)}{d x^{2}} \\
& =\frac{-(1+\mu) d^{2}}{8 k} \frac{d^{2} w_{b} x}{d x^{2}}
\end{aligned}
$$

The differential equation of equilibrium in the $z$ direction is:

$$
\frac{\partial \tau_{z x}}{\partial x}+\frac{\partial \tau_{z y}}{\partial y}+\frac{\partial \sigma_{z z}}{\partial z}+f_{z}=0
$$

where, $f_{z}$ is the body force component in the $z$ direction.

For the thick beam problem, the equilibrium equation is:

$$
\frac{\partial \tau_{z x}}{\partial x}+f_{z}=0
$$

Hence,

$$
\begin{gathered}
\iint_{R^{2}}\left(\frac{\partial \tau_{z x}}{\partial x}+f_{z}\right) d y d z=0 \\
\frac{\partial}{\partial x} \iint_{R^{2}} \tau_{z x} d y d z+\iint_{R^{2}} f_{z} d y d z=0 \\
\frac{\partial}{\partial x} Q_{x}+q=0
\end{gathered}
$$

\subsection{Stability equation}

The stability equation is given by:

$$
\begin{gathered}
N_{x} \frac{d w(x)}{d x}+\frac{d}{d x} M_{x x}-Q_{x}=0 \\
\frac{d Q_{x}}{d x}=0 \text { if } q=0 \\
N_{x} \frac{d}{d x}\left(w_{b}(x)+w_{s}(x)\right)+\frac{d}{d x}\left(-E I \frac{d^{2} w_{b}(x)}{d x^{2}}\right)-Q_{x}=0
\end{gathered}
$$


For prismatic, homogeneous beams,

$$
\begin{gathered}
N_{x}\left(\frac{d}{d x} w_{b}(x)+\frac{d w_{s}(x)}{d x}\right)-E I \frac{d^{3} w_{b}(x)}{d x^{3}}-Q_{x}=0 \\
Q_{x}=N_{x}\left(\frac{d w_{b}(x)}{d x}+\frac{d w_{s}(x)}{d x}\right)-E I \frac{d^{3} w_{b}(x)}{d x^{3}}
\end{gathered}
$$

Differentiation yields:

$$
\frac{d Q_{x}}{d x}=\frac{d}{d x}\left\{N_{x}\left(\frac{d w_{b}(x)}{d x}+\frac{d w_{s}(x)}{d x}\right)-E I \frac{d^{3} w_{b}(x)}{d x^{3}}\right\}
$$

From Eq. (48) we have

$$
\begin{gathered}
\frac{d}{d x}\left\{N_{x}\left(\frac{d w_{b}(x)}{d x}+\frac{d w_{s}(x)}{d x}\right)-E I \frac{d^{3} w_{b}(x)}{d x^{3}}\right\}=-q(x) \\
N_{x}\left(\frac{d^{2} w_{b}(x)}{d x^{2}}+\frac{d^{2} w_{s}(x)}{d x^{2}}\right)-E I \frac{d^{4} w_{b}(x)}{d x^{4}}=-q(x)
\end{gathered}
$$

Using Eqns. (36) and (39) for $w_{s}(x)$ in Eq. (52) we obtain the fourth order ordinary differential equations:

$$
E I \frac{d^{4} w_{b}(x)}{d x^{4}}-N_{x} \frac{d^{2} w_{b}(x)}{d x^{2}}-N_{x} \frac{d^{2}}{d x^{2}}\left(-\frac{h^{2}(1+\mu)}{6 k} \frac{d^{2} w_{b}(x)}{d x^{2}}\right)=q
$$

for rectangular cross-sections and,

$$
E I \frac{d^{4} w_{b}(x)}{d x^{4}}-N_{x} \frac{d^{2} w_{b}(x)}{d x^{2}}-N_{x} \frac{d^{2}}{d x^{2}}\left(\frac{-d^{2}(1+\mu)}{8 k} \frac{d^{2} w_{b}(x)}{d x^{2}}\right)=q
$$

for circular cross-sections.

Further simplifications yield:

$$
\begin{aligned}
& E I \frac{d^{4} w_{b}(x)}{d x^{4}}+\frac{N_{x} h^{2}(1+\mu)}{5} \frac{d^{4} w_{b}(x)}{d x^{4}}-N_{x} \frac{d^{2} w_{b}(x)}{d x^{2}}=q(x) \\
& E I \frac{d^{4} w_{b}(x)}{d x^{4}}+\frac{N_{x} d^{2}(1+\mu)}{8 k} \frac{d^{4} w_{b}(x)}{d x^{4}}-N_{x} \frac{d^{2} w_{b}(x)}{d x^{2}}=q(x)
\end{aligned}
$$

where, $N_{x}$ is tensile.

For axial compressive force $P_{x}$ we have $P_{x}=-N_{x}$, and we obtain the governing equations as:

$$
\left(E I-\frac{P_{x} h^{2}(1+\mu)}{5}\right) \frac{d^{4} w_{b}(x)}{d x^{4}}+P_{x} \frac{d^{2} w_{b}(x)}{d x^{2}}=q(x)
$$

for rectangular cross-sections and

$$
\left(E I-\frac{P_{x} d^{2}(1+\mu)}{8 k}\right) \frac{d^{4} w_{b}(x)}{d x^{4}}+P_{x} \frac{d^{2} w_{b}(x)}{d x^{2}}=q(x)
$$

for circular cross-sections.

For elastic buckling problems where $q(x)=0$, the governing equations are:

$$
\begin{aligned}
& \left(E I-\frac{P_{x} h^{2}(1+\mu)}{5}\right) \frac{d^{4} w_{b}(x)}{d x^{4}}+P_{x} \frac{d^{2} w_{b}(x)}{d x^{2}}=0 \\
& \left(E I-\frac{P_{x} d^{2}(1+\mu)}{8 k}\right) \frac{d^{4} w_{b}(x)}{d x^{4}}+P_{x} \frac{d^{2} w_{b}(x)}{d x^{2}}=0
\end{aligned}
$$

\section{RESULTS}

\subsection{Solution}

We apply the classical method of trial functions to solve the fourth order homogeneous ordinary differential equation (ODE) - Equation (60) - for the buckling of first order shear deformable beam under arbitrary end support conditions. The equation to be solved is expressed as:

$$
\frac{d^{4} w_{b}(x)}{d x^{4}}+\left(\frac{P_{x}}{E I-\frac{P_{x} h^{2}(1+\mu)}{5}}\right) \frac{d^{2} w_{b}(x)}{d x^{2}}=0
$$

Or,

$$
\frac{d^{4} w_{b}(x)}{d x^{4}}+\alpha^{2} \frac{d^{2} w_{b}(x)}{d x^{2}}=0
$$

where,

$$
\alpha^{2}=\frac{P_{x}}{E I-\frac{P_{x} h^{2}(1+\mu)}{5}}
$$

We assume a trial function for $w_{b}(x)$ in exponential form as:

$$
w_{b}(x)=\exp \lambda x
$$

where, $\lambda$ is an unknown parameter of the trial solution we seek to determine.

Then the governing equation becomes:

$$
\lambda^{4} \exp \lambda x+\alpha^{2} \lambda^{2} \exp \lambda x=\left(\lambda^{4}+\alpha^{2} \lambda^{2}\right) \exp \lambda x=0
$$

For non trivial solutions, $\exp \lambda x \neq 0$

The characteristic (auxiliary) polynomial is the fourth order equation:

$$
\lambda^{4}+\alpha^{2} \lambda^{2}=0
$$

Factorising,

$$
\lambda^{2}\left(\lambda^{2}+\alpha^{2}\right)=0
$$

The roots are:

$$
\begin{gathered}
\lambda=0 \text { (twice) } \\
\lambda= \pm i \alpha
\end{gathered}
$$


The basis of solutions is:

$$
\begin{array}{ll}
w_{b}(x)=1, & w_{b}(x)=x, \\
w_{b}(x)=\exp i \alpha x, & w_{b}(x)=\exp (-i \alpha x)
\end{array}
$$

The general solution is then:

$$
w_{b}(x)=c_{1}+c_{2} x+c_{3} \exp (i \alpha x)+c_{4} \exp (-i \alpha x)
$$

where, $c_{1}, c_{2}, c_{3}, c_{4}$ are the four integration constants.

Using the Euler formula, the general solution is:

$$
w_{b}(x)=a_{1}+a_{2} x+a_{3} \cos \alpha x+a_{4} \sin \alpha x
$$

where, $a_{1}, a_{2}, a_{3}$ and $a_{4}$ are four integration constants which are found from the end support conditions.

\subsection{Solution for simply supported ends for first order shear} deformable beam with simply supported ends $(x=0, x=l)$

The boundary conditions for the first order shear deformable beam with simple supports at $\mathrm{x}=0$ and $\mathrm{x}=1$ as shown in Figure 2 are:

$$
\begin{gathered}
w_{b}(x=0)=0 ; w_{b}^{\prime \prime}(x=0)=0 ; w_{b}(x=l)=0 ; w_{b}^{\prime \prime}(x=l)=0 \\
w_{b}^{\prime \prime}(x)=-a_{3} \alpha^{2} \cos \alpha x-a_{4} \alpha^{2} \sin \alpha x
\end{gathered}
$$

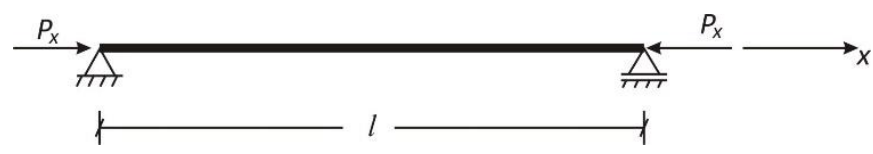

Figure 2. First order shear deformable beam with simple supports at $x=0, x=l$

Using the boundary conditions, we have the homogeneous equation:

$$
\left(\begin{array}{cccc}
1 & 0 & 1 & 0 \\
0 & 0 & -\alpha^{2} & 0 \\
1 & l & \cos \alpha l & \sin \alpha l \\
0 & 0 & -\alpha^{2} \cos \alpha l & -\alpha^{2} \sin \alpha l
\end{array}\right)\left(\begin{array}{l}
a_{1} \\
a_{2} \\
a_{3} \\
a_{4}
\end{array}\right)=\left(\begin{array}{l}
0 \\
0 \\
0 \\
0
\end{array}\right)
$$

The characteristic buckling equation is

$$
\left|\begin{array}{cccc}
1 & 0 & 1 & 0 \\
0 & 0 & -\alpha^{2} & 0 \\
1 & l & \cos \alpha l & \sin \alpha l \\
0 & 0 & -\alpha^{2} \cos \alpha l & -\alpha^{2} \sin \alpha l
\end{array}\right|=0
$$

Expansion of the determinant yields the characteristic buckling equation as:

$$
\sin \alpha l=0
$$

Solving,

$$
\alpha l=\sin ^{-1} 0=m \pi ; \quad m=1,3,5,7,9,11, \ldots
$$

$$
\alpha=\frac{m \pi}{l}
$$

Hence,

$$
\alpha^{2}=\frac{P_{x}}{E I-\frac{P_{x} h^{2}(1+\mu)}{5}}=\left(\frac{m \pi}{l}\right)^{2}
$$

\begin{tabular}{|c|c|c|c|}
\hline$h / l$ & $\begin{array}{c}\left(\times E \mathbf{E} / \mathbf{l}^{2}\right) \\
P_{x_{c r}}(\text { for } \mu=0.25)\end{array}$ & $\begin{array}{c}\left(\times E \boldsymbol{I} / \mathbf{l}^{2}\right) \\
P_{x_{c r}}(\text { for } \mu=0.30)\end{array}$ & $\begin{array}{c}\text { Pakhare et al. } \\
{[2]} \\
P_{x_{c r}}(\mu=0.30)\end{array}$ \\
\hline 0.01 & 9.86717 & 9.8671 & 9.8671 \\
\hline 0.02 & 9.85987 & 9.8595 & 9.8595 \\
\hline 0.05 & 9.8091 & 9.8067 & 9.8067 \\
\hline 0.10 & 9.63195 & 9.6227 & 9.6227 \\
\hline 0.15 & 9.35050 & 9.3309 & 9.3309 \\
\hline 0.20 & 8.98302 & 8.9509 & 8.9509 \\
\hline 0.25 & 8.55094 & 8.5055 & 8.5055 \\
\hline 0.30 & 8.07616 & 8.0179 & 8.0179 \\
\hline 0.35 & 7.57885 & 7.5091 & 7.5091 \\
\hline 0.40 & 7.07608 & 6.9969 & 6.9969 \\
\hline 0.45 & 6.58128 & 6.49472 & \\
\hline 0.50 & 6.10422 & 6.01246 & \\
\hline
\end{tabular}

$$
\begin{gathered}
P_{x}=\left(\frac{m \pi}{l}\right)^{2}\left(E I-\frac{P_{x} h^{2}(1+\mu)}{5}\right) \\
P_{x}\left(1+\frac{h^{2}(1+\mu)}{5}\left(\frac{m \pi}{l}\right)^{2}\right)=\left(\frac{m \pi}{l}\right)^{2} E I \\
P_{x}=\frac{\left(\frac{m \pi}{l}\right)^{2} E I}{1+\frac{h^{2}(1+\mu)}{5}\left(\frac{m \pi}{l}\right)^{2}}=\left(\frac{(m \pi)^{2}}{\left.1+\frac{h^{2}(1+\mu)}{5}\left(\frac{m \pi}{l}\right)^{2}\right) \frac{E I}{l^{2}}}\right. \\
P_{x_{c r}}=P_{x}(m=1)=\left(\frac{\pi^{2}}{1+\frac{\pi^{2}(1+\mu)}{5} \frac{h^{2}}{l^{2}}}\right) \frac{E I}{l^{2}}
\end{gathered}
$$

$P_{x_{c r}}$ is tabulated for $\mu=0.25$, and $\mu=0.30$ as shown in Table 1 for various values of beam depth to span ratio $(h / l)$

Table 1. Exact critical buckling load for first order shear deformable beam for various ratios of $h / l$ and for $\mu=0.25$ and $\mu=0.30$ (rectangular cross-sections)

3.3 Solution for fixed pinned ends for a first order shear deformable beam fixed at $x=0$, and pinned at $x=l$

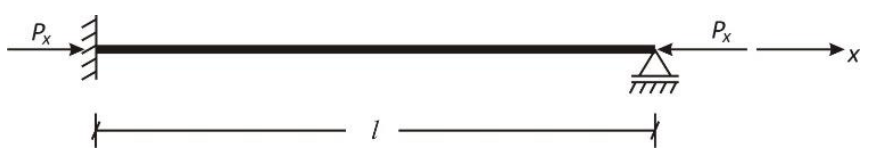

Figure 3. First order shear deformable beam fixed at $x=0$, and pinned at $x=1$

The boundary conditions for the shear deformable beam with support conditions shown in Figure 3 are:

$$
w_{b}(x=0)=0 ; w_{b}^{\prime}(x=0)=0 ; w_{b}(x=l)=0 ; w_{b}^{\prime \prime}(x=l)=0
$$


Enforcement of boundary conditions yield the system of homogeneous algebraic equations:

$$
\left(\begin{array}{cccc}
1 & 0 & 1 & 0 \\
0 & 1 & 0 & \alpha \\
1 & l & \cos \alpha l & \sin \alpha l \\
0 & 0 & \cos \alpha l & \sin \alpha l
\end{array}\right)\left(\begin{array}{l}
a_{1} \\
a_{2} \\
a_{3} \\
a_{4}
\end{array}\right)=\left(\begin{array}{l}
0 \\
0 \\
0 \\
0
\end{array}\right)
$$

For nontrivial solutions,

$$
\left|\begin{array}{cccc}
1 & 0 & 1 & 0 \\
0 & 1 & 0 & \alpha \\
1 & l & \cos \alpha l & \sin \alpha l \\
0 & 0 & \cos \alpha l & \sin \alpha l
\end{array}\right|=0
$$

Expansion of the determinant yields the characteristic buckling equation as the transcendental equation:

$$
\tan (\alpha l)-\alpha l=0
$$

Using software, or iterative methods - Newton Raphson, Regula-Falsi, simple iteration, the first four nontrivial roots of the transcendental equation are:

$$
\begin{gathered}
\alpha_{1} l= \pm 4.49341 \\
\alpha_{2} l= \pm 7.72525184 \\
\alpha_{3} l= \pm 10.9041266 \\
\alpha_{4} l= \pm 14.0661939
\end{gathered}
$$

Each root can be used to compute a buckling load.

The smallest root yields the critical buckling load. The critical buckling load $P_{x_{c r}}$ is found as:

$$
\alpha_{1}^{2}=\left(\frac{4.49341}{l}\right)^{2}=\frac{P_{x c r}}{E I-\frac{P_{x c r} h^{2}(1+\mu)}{5}}
$$

Solving for $P_{x_{c r}}$,

$$
P_{x c r}=\frac{20.19073}{1+4.038146(1+\mu) \frac{h^{2}}{l^{2}}} \frac{E I}{l^{2}}
$$

For $\mu=0.25$ :

$$
P_{x_{c r}}(\mu=0.25)=\frac{20.19073}{1+5.04768 \frac{h^{2}}{l^{2}}} \frac{E I}{l^{2}}
$$

For $\mu=0.30$ :

$$
P_{x_{c r}}(\mu=0.30)=\frac{20.19073}{1+5.2495898 \frac{h^{2}}{l^{2}}} \frac{E I}{l^{2}}
$$

The critical buckling load is calculated and tabulated in Table 2 for various values of the ratio $h / l$ for $\mu=0.25$ and $\mu=$ 0.30

Table 2. Exact critical buckling loads for shear deformable beam fixed at $x=0$, pinned at $x=l$ for various ratios of $h / l$ and for $\mu=0.25, \mu=0.30$ (rectangular cross-sections)

\begin{tabular}{ccc}
\hline \multicolumn{1}{l}{$h / l$} & $P_{x c r}\left(\mu=0.25 \times \frac{E I}{l^{2}}\right)$ & $P_{x c r}(\mu=0.30) \times \frac{E I}{l^{2}}$ \\
\hline 0.01 & 20.180544 & 20.180136 \\
0.02 & 20.150045 & 20.148422 \\
0.05 & 19.939111 & 19.929180 \\
0.10 & 19.220539 & 19.183666 \\
0.15 & 18.131486 & 18.057817 \\
0.20 & 16.798909 & 16.68678 \\
0.25 & 15.348565 & 15.202725 \\
0.30 & 13.883554 & 13.712215 \\
0.35 & 12.476192 & 12.288382 \\
0.40 & 11.169732 & 10.973614 \\
0.45 & 9.984758 & 9.786873 \\
0.50 & 8.926368 & 8.731514 \\
\hline
\end{tabular}

\subsection{Solution for fixed-fixed ends}

A shear deformable beam of length $l$ fixed at the two ends $x=0$, and $x=l$ as shown in Figure 4, is considered.

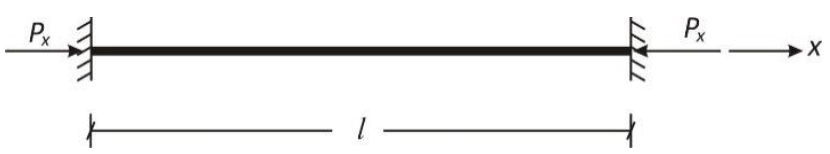

Figure 4. Shear deformable beam with fixed ends ( $x=0, x=l)$

The boundary conditions are:

$w_{b}(x=0)=0 ; w_{b}^{\prime}(x=0)=0 ; w_{b}(x=l)=0 ; w_{b}^{\prime}(x=l)=0$

Enforcement of the boundary conditions yield the homogeneous algebraic equations given by:

$$
\left(\begin{array}{cccc}
1 & 0 & 1 & 0 \\
0 & 1 & 0 & \alpha \\
1 & l & \cos \alpha l & \sin \alpha l \\
0 & 1 & -\alpha \cos \alpha l & \alpha \cos \alpha l
\end{array}\right)\left(\begin{array}{l}
a_{1} \\
a_{2} \\
a_{3} \\
a_{4}
\end{array}\right)=\left(\begin{array}{l}
0 \\
0 \\
0 \\
0
\end{array}\right)
$$

For nontrivial solutions, the characteristic buckling equation is found from the determinantal equation:

$$
\left|\begin{array}{cccc}
1 & 0 & 1 & 0 \\
0 & 1 & 0 & \alpha \\
1 & l & \cos \alpha l & \sin \alpha l \\
0 & 1 & -\alpha \sin \alpha l & \alpha \cos \alpha l
\end{array}\right|=0
$$

Expansion of the determinant yields the transcendental equation:

$$
\alpha l \sin \alpha l+2 \cos \alpha l-2=0
$$

The first four nontrivial roots of the transcendental equation are found using software or iterative methods - NewtonRaphson, simple iteration, Regula-Falsi as: 


$$
\begin{aligned}
& \alpha_{1} l= \pm 6.28318530717959 \\
& \alpha_{2} l= \pm 8.98681891581813 \\
& \alpha_{3} l= \pm 12.5663706143592 \\
& \alpha_{4} l= \pm 15.4505036738754
\end{aligned}
$$

The critical buckling load is found from the smallest nontrivial root, $\alpha_{1}$. Thus,

$$
\begin{gathered}
\alpha_{1}^{2}=\left(\frac{6.283185}{l}\right)^{2}=\frac{P_{x c r}}{E I-\frac{P_{x c r}(1+\mu) h^{2}}{5}} \\
P_{x c r}=\frac{39.478414}{l^{2}}\left(E I-\frac{P_{x c r}(1+\mu) h^{2}}{5}\right)
\end{gathered}
$$

Solving for $P_{x c r}$,

$$
P_{x c r}=\frac{39.478414}{1+7.89568 \frac{(1+\mu) h^{2}}{l^{2}}} \frac{E I}{l^{2}}
$$

For $\mu=0.25$ :

$$
P_{x_{c r}}(\mu=0.25)=\frac{39.478414}{1+9.8696 \frac{h^{2}}{l^{2}}} \frac{E I}{l^{2}}
$$

For $\mu=0.30$ :

$$
P_{x_{c r}}(\mu=0.30)=\frac{39.478414}{1+10.264384 \frac{h^{2}}{l^{2}}} \frac{E I}{l^{2}}
$$

The critical buckling load is calculated and presented in Table 3 for various values of the ratio $h / l$ for $\mu=0.25$ and $\mu=$ 0.30 .

Table 3. Exact critical buckling load for first order shear deformable beam with fixed-fixed ends for various values of $h / l$ and for $\mu=0.25$ and $\mu=0.30$

\begin{tabular}{ccc}
\hline$h / l$ & $P_{x c r}(\mu=0.25) \times \frac{E I}{l^{2}}$ & $P_{x c r}(\mu=0.30) \times \frac{E I}{l^{2}}$ \\
\hline 0.01 & 39.43949 & 39.43793 \\
0.02 & 39.32317 & 39.31699 \\
0.05 & 38.52778 & 38.49071 \\
0.10 & 35.93206 & 35.80341 \\
0.15 & 32.30465 & 32.07154 \\
0.20 & 28.30432 & 27.98745 \\
0.25 & 24.41687 & 24.04985 \\
0.30 & 20.90725 & 20.52112 \\
0.35 & 17.87141 & 17.48854 \\
0.40 & 15.30684 & 14.94092 \\
0.45 & 13.16564 & 12.82376 \\
0.50 & 11.38560 & 11.07049 \\
\hline
\end{tabular}

\subsection{Solution for pinned-fixed ends}

For a shear deformable beam of length $l$ pinned at $x=0$ and fixed at $x=l$, as shown in Figure 5 , the boundary conditions are:

$$
\begin{gathered}
w_{b}(x=0)=0 ; w_{b}^{\prime \prime}(x=0)=0 ; \\
w_{b}(x=l)=0 ; w_{b}^{\prime}(x=l)=0
\end{gathered}
$$

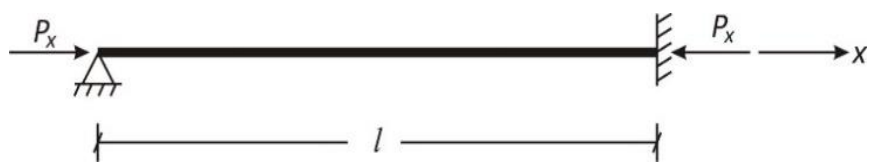

Figure 5. Elastic buckling of shear deformable beam with pinned-fixed ends

Enforcement of boundary conditions yield:

$$
\left(\begin{array}{cccc}
1 & 0 & 1 & 0 \\
0 & 0 & -\alpha^{2} & 0 \\
1 & l & \cos \alpha l & \sin \alpha l \\
0 & 1 & -\alpha \sin \alpha l & \alpha \cos \alpha l
\end{array}\right)\left(\begin{array}{l}
a_{1} \\
a_{2} \\
a_{3} \\
a_{4}
\end{array}\right)=\left(\begin{array}{l}
0 \\
0 \\
0 \\
0
\end{array}\right)
$$

For nontrivial solutions, we obtain determinantal equation:

$$
\left|\begin{array}{cccc}
1 & 0 & 1 & 0 \\
0 & 0 & -\alpha^{2} & 0 \\
1 & l & \cos \alpha l & \sin \alpha l \\
0 & 1 & -\alpha \sin \alpha l & \alpha \cos \alpha l
\end{array}\right|=0
$$

Expansion of the determinant yields the characteristic buckling equation as Eq. (88). This is the same result obtained for first order shear deformable beams fixed at $x=0$ and pinned at $x=l$. The buckling loads are the same as obtained for fixed-pinned ends and the critical buckling load for shear deformable beams pinned at $x=0$, and fixed at $x=l$ for various ratios of $h / l$ and for $\mu=0.25$, and $\mu=0.30$ for rectangular cross sections are the same as shown in Table 2 for fixed-pinned ends.

\section{DISCUSSION}

In this work, the elastic buckling problem of shear deformable beams has been presented from first principles as a boundary value problem of the mathematical theory of elasticity. The formulation presented is for a thick beam or a moderately thick beam with longitudinal coordinate axis on the $x$-axis and the cross-sectional plane on the $y z$ plane. The beam material is assumed homogeneous, isotropic and linear elastic and the formulation was based on small deformation assumptions of classical elasticity theory. Based on displacement field assumptions given by Eqns. (1-3), where the rotation of the cross-section at the neutral axis is given by Eq. (4) the strains and stress fields were found from the kinematic relations and the constitutive laws as Eqns. (6-11) and (12-13) respectively. The stress resultants were obtained as Eqns. (15) and (18) for the bending moment and shear force respectively. The differential equations of equilibrium expressed in terms of the stress resultants were used to obtain 
the governing boundary value problem as the fourth order differential equation given by Eq. (57) for rectangular crosssectional shapes and Eq. (58) for circular cross-sections.

The method of trial functions was used to solve the homogeneous fourth order ordinary differential equation (ODE) governing the elastic buckling of shear deformable beam when transverse forces are ignored and the beam is subject to only axial compression. The assumption of a trial solution for the buckled shape of the beam in the form of the exponential function given by Eq. (64) reduced the fourth order ODE to an algebraic problem described for nontrivial solutions by the characteristic (auxiliary) polynomial given by the fourth degree polynomial in Eq. (66). The four roots (zeros) of the characteristic equation are obtained by factorisation as Eqns. (68) and (69). The zeros of the characteristic polynomial yielded the solution basis functions as Eq. (70). The general solution is then obtained in terms of exponential functions as Eq. (71), and in terms of trigonometric functions as Eq. (72). The general solution is obtained in terms of four integration constants which are determined only if the boundary conditions, determined by the support conditions are specified.

Specific cases of end support namely: (i) both ends simply supported; (ii) fixed pinned ends; (iii) fixed - fixed ends, and (iv) pinned - fixed ends were considered, and analytical solutions were presented for each of the cases. For simply supported ends, the boundary conditions are given by Equation (73). Enforcement of the boundary conditions for simply supported ends led to the system of homogeneous algebraic equations in terms of the unknowns, given by Eq. (75). For nontrivial solutions, the determinant of the coefficient matrix would vanish yielding the characteristic buckling equation as Eq. (76). The expansion of the determinant yielded Eq. (77) which was solved to obtain Eq. (79) which has an infinite number of roots. The buckling loads were then obtained for simply supported ends as Eq. (83) and the critical buckling load found as Eq. (84). The critical buckling load was determined for values of Poisson's ratio $\mu=0.25$ and $\mu=0.30$, and for various values of $h / l$ and presented in Table 1 . From Table 1, it is observed that for both values of Poisson's ratio, as $h / l$ increases, the critical buckling load coefficient decreases. For small values of $h / l$ corresponding to the range of values of $h / l$ which define thin (slender) beams, the critical buckling load coefficient is approximately the same as the critical buckling load coefficient of Euler - Bernoulli beams with simply supported ends.

For shear deformable beams with fixed - pinned ends, the boundary conditions are given by Eq. (85). Enforcement of the boundary conditions yielded the system of algebraic equation - Eq. (87), which upon expansion yielded the transcendental equation - Eq. (88), which has an infinite number of roots. The zeros of the transcendental equation for the first four roots are found as Eqns. (89-92), with each root corresponding to a buckling load. The smallest root was used to compute the critical buckling load as Eq. (94), for any value of $\mu$, and Eq. (95) for $\mu=0.25$ and Eq. (96) for $\mu=0.30$.

Critical buckling loads for first order shear deformable beam with fixed - pinned ends for various values of $h / l$ and for $\mu=0.25$ and $\mu=0.30$ were computed as shown in Table 2 . The elastic buckling problem of first order shear deformable beam with fixed - fixed ends was considered with the boundary conditions as Eq. (97). Enforcement of the boundary conditions resulted in the homogeneous algebraic problem in Eq. (98), which gave the characteristic buckling equation for nontrivial solution as Eq. (99). Expansion of the determinant yielded the characteristic buckling equation as the transcendental equation - Eq. (100) - with an infinite number of roots. The first four zeros of the buckling equation were found as Eqns. $(101-104)$ and can be used to find the first four buckling loads. The least nontrivial root gave the critical buckling load as Eq. (107), for any value of Poisson ratio, and Equations (108) and (109) for $\mu=0.25$ and $\mu=0.30$ respectively. Critical buckling loads for fixed-fixed ends were calculated and shown in Table 3. For pinned - fixed ends, the boundary conditions are given as Eq. (110). The use of the boundary conditions gave rise to the homogeneous algebraic equations in Eq. (111). The requirement of nontrivial solutions of the algebraic problem yielded the characteristic equation shown in Eq. (112).

Expansion of the determinant yielded the characteristic buckling equation as Eq. (88), which was solved to obtain the zeros as Eqns. (89-92); the roots were found to be infinite in number and the buckling loads were found as Eq. (94). The critical buckling load was found as Eqns. (95), (120) for $\mu=$ 0.25 and (96) for $\mu=0.30$. The critical buckling loads were computed for various values of $h / l$ and $\mu=0.25, \mu=0.30$, and presented in Table 2.

From Tables 1, 2, and 3, it is observed that as $h / l<0.02$ the critical buckling load coefficients obtained for the various end support conditions are approximately equal to the critical buckling load coefficients for the Euler - Bernoulli beam with the corresponding end support conditions. As $h / l>0.02$, which is the threshold for defining slender beams, the critical buckling load coefficient obtained becomes smaller than the critical buckling load obtained using the Euler-Bernoulli beam theory. For $h / l>0.02$, the effect of shear deformation is observed to significantly affect the critical buckling load of the beam. Hence shear deformation effects need to be considered for a more realistic analysis of the critical buckling load capacities of moderately thick and thick beams for a safe design of such structures.

\section{CONCLUSION}

The conclusions of the study are as follows:

(i) The elastic buckling problem of shear deformable thick and moderately thick beams has been formulated from first principles as a boundary value problem of the mathematical theory of elasticity.

(ii) The problem satisfied the kinematic relations, the constitutive laws and the differential equations of equilibrium.

(iii) The boundary value problem was obtained in general as a fourth order non homogeneous ordinary linear differential equation when there are transversely applied loads in addition to the axial compressive load, and a fourth order homogeneous ODE when there is only axial compressive load, and no transverse load.

(iv) The method of trial function for the case when no transverse force is considered transformed the BVP to an algebraic equation, which is solved to obtain the characteristic buckling equation found as a fourth degree polynomial whose roots yielded the solution basis functions, and led to obtaining the general solutions in terms of four unknown constants of integration.

(v) Boundary conditions for the specific cases of end supports were used in obtaining the characteristic buckling equations, which were solved to obtain the buckling loads. 
(vi) For $h / l<0.02$ which defines slender (thin) beams, the critical buckling load coefficients obtained for the various end support conditions are approximately equal to the critical buckling load coefficients for the Euler - Bernoulli beam with the corresponding end support conditions.

(vii) As $h / l>0.02$ which is the threshold for thin beams, the critical buckling load coefficient obtained for moderately thick beams and thick beams is smaller than the critical buckling load from the Euler - Bernoulli beam theory.

(viii) For $h / l>0.02$ shear deformation effect is found to significantly reduce the critical buckling load of beams.

(ix) Shear deformation effects need to be considered for a more realistic analysis of the critical buckling load capacities of moderately thick and thick beams for safety in their design.

\section{REFERENCES}

[1] Sayyad, A.S., Ghugal, Y.M. (2016). Single variable refined beam theories for the bending, buckling and free vibration of homogeneous beams. Applied and Computational Mechanics, 10: 123-138.

[2] Pakhare, K.S., Shimpi, R.P., Guruprasad, P.J. (2017). Buckling analysis of thick isotropic shear deformable beams. International Conference on Theoretical, Applied, Computational and Experimental Mechanics, 2017 IIT Kharagpur India, pp. 1-7.

[3] Ike, C.C. (2018). Fourier sine transform method for the free vibration of Euler - Bernoulli beam resting on Winkler foundation. International Journal of Darshan Institute on Engineering Research and Emerging Technologies (IJDI-ERET), 7(1): 1-6. https://doi.org/10.32692/IJDI-ERET/7.1.2018.1801

[4] Ike, C.C. (2018). Point collocation method for the analysis of Euler - Bernoulli beam on Winkler foundation. International Journal of Darshan Institute on Engineering Research and Emerging Technologies (IJDI-ERET), 7(2): 1-7.

[5] Onyia, M.E., Rowland-Lato, E.O. (2018). Determination of the critical buckling load of shear deformable unified beam. International Journal of Engineering and Technology (IJET), $10(3)$ : $\quad 647-657$. https://doi.org/10.21817/ijet/2018/v10i3/181003026

[6] Pakhare, K.S., Mitra, M., Shimpi, R.P. (2016). Development of single variable new first - order shear deformation theories for plates and beams. Master of Technology Dissertation. Department of Aerospace Engineering, Indian Institute of Technology Bombay.

[7] Ghugal, Y.M. (2007). A single variable parabolic shear deformation theory for flexure and flexural vibration of thick isotropic beams. Proceedings of the Third International Conference on Structural Engineering Mechanics and Computation, Cape Town South Africa, pp. 77-78.

[8] Ghugal, Y.M., Shimpi, R.P. (2000). A trigonometric shear deformation theory for flexure and free vibration of isotropic thick beams. Proceeding of Structural Engineering Convention (SPC 2000) IIT Bombay, Mumbai, India, pp. 255-263.

[9] Sayyad, A.S. (2011). Comparison of various refined beam theories for the bending and free vibration analysis of thick beams. Applied and Computational Mechanics, 5(2): 217-230.

[10] Ghugal, Y.M. (2006). A new refined bending theory for thick beam including transverse shear and transverse normal strain effects. Departmental Report Applied Mechanics Department Government College of Engineering Anrangabad India, 1-96.

[11] Levinson, M. (1981). A new rectangular beam theory. Journal of Sound and Vibration, 74(1): 81-87. https://doi.org/10.1016/0022-460X(81)90493-4

[12] Krishna Murty, A.V. (1984). Towards a consistent beam theory. AIAA Journal, 22(6): 811-816. https://doi.org/10.2514/3.8685

[13] Heyliger, P.R., Reddy, J.N. (1988). A higher order beam finite element for bending and vibration problems. Journal of Sound and Vibration, 126(2): 309-326. https://doi.org/10.1016/0022-460X(88)90244-1

[14] Sayyad, A.S., Ghugal, Y.M. (2011). Effect of transverse shear and transverse normal strain on the bending analysis of cross-ply laminated beams. International Journal of Applied Mathematics and Mechanics, 7(12): 85-118.

[15] Soldatos, K.P. (1992). A transverse shear deformation theory for homogeneous monoclinic plates. Acta Mechanics, 94(3-4): 195-200.

[16] Ghugal, Y.M., Sharma, P.V. (2009). A hyperbolic shear deformation theory for flexure and vibration of thick isotropic beams. International Journal of Computational Methods, 6(4): 585-604. https://doi.org/10.1142/S0219876209002017

[17] Karama, M., Abaq, K.S., Mistou, S. (2003). Mechanical behaviour of laminated composite beam by new multilayered laminated composite structures model with transverse shear stress continuity. International Journal of Solids and Structures, 40: 1523-1546. https://doi.org/10.1016/S0020-7683(02)00647-9

[18] Sayyad, A.S., Ghugal, Y.M. (2017). A unified shear deformation theory for the bending of isotropic, functionally graded, laminated and sandwich beams and plates. International Journal of Applied Mechanics, 9(1): 1750007. https://doi.org/10.1142/S1758825/117500077

\section{NOMENCLATURE}

$\begin{array}{ll}x, y, z & \begin{array}{l}\text { Cartesian coordinates } \\ \text { beam length }\end{array} \\ b & \text { beam width } \\ h & \text { beam depth (thickness) } \\ x & \begin{array}{l}\text { longitudinal coordinate } \\ \text { cross-sectional coordinates }\end{array} \\ y z & \text { inplane displacement field } \\ u & \text { transverse displacement field } \\ w & \text { transverse displacement field } \\ w & \text { flexural component of transverse displacement } \\ w_{b} & \text { field } \\ & \text { shear component of transverse displacement field } \\ w_{s} & y \text { component of displacement field } \\ v & \text { rotation of the cross-section at the neutral axis } \\ \varphi(x) & \text { normal strains } \\ \varepsilon_{x x}, \varepsilon_{y y}, \varepsilon_{z z} & \text { shear strains } \\ \gamma_{x y}, \gamma_{x z}, \gamma_{y z} & \text { normal stresses } \\ \sigma_{x x}, \sigma_{y y}, \sigma_{z z} & \text { shear stresses } \\ \tau_{x y}, \tau_{x z}, \tau_{y z} & \text { Young's modulus of elasticity } \\ E & \text { shear modulus or modulus of rigidity } \\ G & \text { Poisson's ratio } \\ \mu & \text { bending moment } \\ M_{x x} & \end{array}$




$\begin{array}{ll}Q_{x} & \text { shear force } \\ R^{2} & \text { two-dimensional region of integration } \\ I & \text { moment of inertia } \\ k & \text { shear correction/modification factor } \\ f_{x} & \text { body force component in the } x \text { direction. } \\ d & \text { diameter of beam of circular cross-section } \\ f_{z} & \text { body force component in the } z \text { direction } \\ N_{x}, P_{x} & \text { axial force on beam } \\ q(x) & \text { transverse force distribution } \\ \alpha^{2} & \text { parameter defined in terms of } P_{x}, h, \mu, E I \\ \lambda & \text { unknown parameter of the trial buckling function } \\ i & \text { imaginary number } \\ \exp & \text { exponential } \\ c_{1}, c_{2}, c_{3}, c_{4} & \text { integration constants } \\ a_{1}, a_{2}, a_{3}, a_{4} & \\ P_{x x_{c r}} & \text { critical buckling load } \\ m & \text { integer } \\ \cos & \text { cosine } \\ \sin & \text { sine } \\ \tan & \text { tangent }\end{array}$

greater than

less than

cross-sectional area

determinant

integral

double integral

ordinary derivative with respect to $x$

ordinary differential equation Euler - Bernoulli beam theory first order shear deformation theory Timoshenko beam theory higher order shear deformation theory unified beam theory boundary value problem three dimensional derivative critical critical buckling load 